\title{
Double burden of nutritional disorders in young Iranian children: findings of a nationwide screening survey
}

\author{
Mohammad E Motlagh, Roya Kelishadi*, Mohammad A Amirkhani, Hasan Ziaoddini, \\ Marziyeh Dashti, Tahereh Aminaee, Gelayol Ardalan, Parisa Mirmoghtadaee, \\ Sousan Keshavarz and Parinaz Poursafa \\ Department of Pediatric Preventive Cardiology, Isfahan Cardiovascular Research Centre, Isfahan University of \\ Medical Sciences, PO Box 81465-1148, Isfahan, Iran
}

Submitted 18 January 2010: Accepted 30 June 2010: First published online 15 September 2010

\begin{abstract}
Objective: To assess the national prevalence of short stature, underweight, overweight and obesity in 6-year-old Iranian children before school entry.

Design: Cross-sectional nationwide survey.

Setting: Mandatory national screening programme before entrance to elementary school in 2008 in Iran.

Subjects: All Iranian children entering public and private elementary schools.

Results: The study population comprised 862433 children ( $48 \cdot 4 \%$ girls, $77 \cdot 2 \%$ urban resident). Overall, $6.5 \%$ of children had short stature, $19 \cdot 1 \%$ were underweight, $12 \cdot 8 \%$ were overweight and $3 \cdot 4 \%$ were obese. There was no significant difference in terms of gender, but considerably larger differences were documented among various provinces.

Conclusions: The double burden of nutritional disorders among young children warrants a multi-faceted national policy with evidence-based local programmes. Such planning needs a comprehensive surveillance system and centralized data registry for children's growth.
\end{abstract}

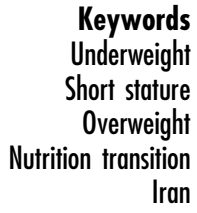

Weight disorders, either underweight or overweight, are worldwide major health public concerns, especially in developing countries ${ }^{(1,2)}$. Moreover, stunting is considered as the most sensitive indicator of malnutrition in developing countries ${ }^{(3)}$. Some variables such as age, sex, frequency of episodes of infection and socio-economic indicators of household poverty are important determinants of short stature ${ }^{(4,5)}$.

While childhood underweight is still one of the major considerations of health policy makers, overweight is becoming increasingly prevalent in developing countries $^{(2,6)}$. The analysis of 160 nationally representative surveys from ninety-four developing countries confirmed this paradox in pre-school-aged children; it showed an increasing prevalence of overweight and obesity from childhood to adulthood, although the rates of early childhood malnutrition remained relatively high ${ }^{(7)}$.

Similar to many other developing countries, in recent decades Iran has been experiencing a rapid phase of epidemiological transition in terms of urbanization and nutrition transition ${ }^{(8)}$. Considering the great diversity in sociodemographic factors in different provinces in Iran, national representative studies may provide a view on these health concerns for health policy makers at national and regional levels.

Most previous studies in Iran have been limited to one city and the information might not be generalizable to other parts of the country. In our previous national survey among 6-18-year-old school students in the provincial counties of twenty-three provinces in Iran, the prevalences of overweight and underweight were nearly similar, i.e. both being about 13\% with significant diversity among different provinces. Of special concern in that study was the higher prevalence of overweight in elementary-school than in high-school students ${ }^{(9,10)}$.

The importance of growth disorders in early childhood necessitated the conduct of a study including a larger sample size of a younger age group living in counties other than the provincial ones included in the aforementioned study. As there is no national centralized registry system for anthropometric measures of pre-school-aged children in Iran, we used the data of the nationwide mandatory screening of all children at entering elementary schools to determine the prevalence of short stature, underweight, overweight and obesity in different provinces with various socio-economic, ethnic and cultural backgrounds. 


\section{Methods}

This nationwide survey was conducted in 2008 as part of the mandatory national screening programme conducted by the Ministry of Health and Medical Education and the Ministry of Education and Training among all children entering elementary schools. All ethical concerns have been considered by these ministries. According to national laws, elementary education is mandatory for all children; thus the study population comprised all children entering public and private elementary schools in Iran.

Training sessions were organized for all health-care providers collaborating with the present survey, and calibrated instruments were used for anthropometric measurements. The Data and Safety Monitoring Board of the screening programme closely supervised the quality control and quality assurance of the survey at the national level.

Weight was measured in light outdoor clothing, to the nearest $0 \cdot 1 \mathrm{~kg}$, and height was measured without shoes, using a portable stadiometer. BMI was calculated as weight $(\mathrm{kg})$ divided by height squared $\left(\mathrm{m}^{2}\right)$. To determine abnormal BMI levels, all measurements were compared to the percentiles on the growth charts of the Centers for Disease Control and Prevention ${ }^{(11)}$; these charts are documented to have close agreement with Iranian charts ${ }^{(9)}$. Underweight, overweight and obesity were considered as age- and gender-specific BMI of $<5$ th, 85th-94th and $\geq 95$ th percentile curves, respectively. Short stature was considered as height $>2$ sD below the corresponding mean height for a given age and sex ${ }^{(12)}$.
The data-checking process was conducted first at the provincial and then at the national level. After editing, the data were analysed using the Statistical Package for Social Sciences statistical software package version $13 \cdot 0$ (SPSS Inc., Chicago, IL, USA). Descriptive analysis was used to determine the prevalence of weight disorders and short stature. Analyses were initially stratified by gender, but as the differences were not significant, the results are presented for girls and boys in combination.

\section{Results}

This national survey comprised 862433 children (48.4\% girls); $77 \cdot 2 \%$ of them lived in urban areas, and $44.9 \%$ of the children were the first child of the family. The children had a mean age of 6.7 (SD 0.7) years $(6 \cdot 6(\mathrm{SD} 0.5)$ years in boys $v .6 .9$ (SD 0.8 ) years in girls, $P=0 \cdot 1$ ); the mean of weight, height and BMI was $20 \cdot 4(\mathrm{SD} 2 \cdot 2) \mathrm{kg}$, $114 \cdot 7(\mathrm{sD} 2 \cdot 8) \mathrm{cm}$ and $15 \cdot 5(\mathrm{sD} 2 \cdot 7) \mathrm{kg} / \mathrm{m}^{2}$, respectively, without significant difference in terms of gender $(21 \cdot 1$ (SD $2 \cdot 6) \mathrm{kg}, 116 \cdot 5(\mathrm{sD} 3 \cdot 4) \mathrm{cm}$ and $15 \cdot 7(\mathrm{sD} 2 \cdot 1) \mathrm{kg} / \mathrm{m}^{2}$ in boys v. $20 \cdot 2(\mathrm{sD} 3 \cdot 1) \mathrm{kg}, 115 \cdot 1(\mathrm{sD} 4 \cdot 7) \mathrm{cm}$ and $15 \cdot 4(\mathrm{sD} 2 \cdot 7) \mathrm{kg} / \mathrm{m}^{2}$ in girls, respectively).

Short stature was documented in $6.5 \%$ of children; the prevalence in each province is presented in Fig. 1. The prevalence of underweight was $19 \cdot 1 \%$, as depicted in Fig. 2; this prevalence had large variations in different provinces.

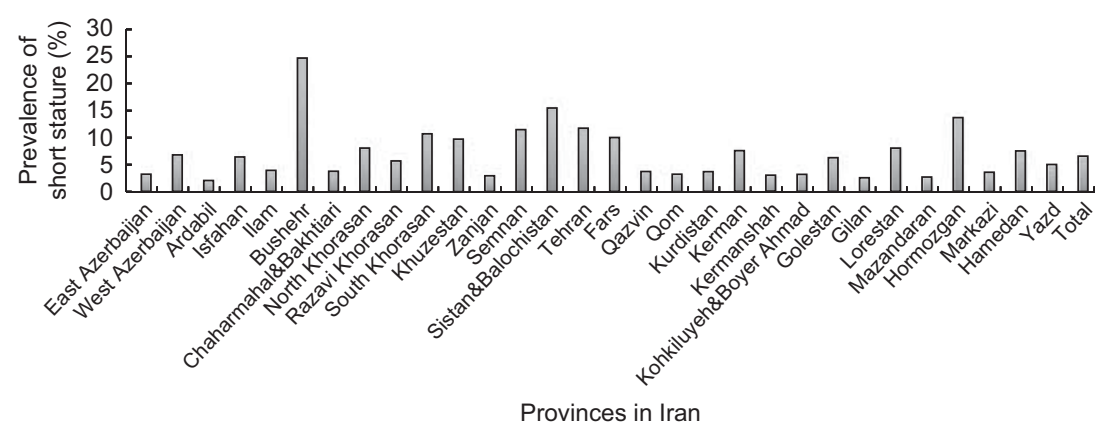

Fig. 1 Prevalence of short stature among 6-year-old children in different provinces in Iran, 2008

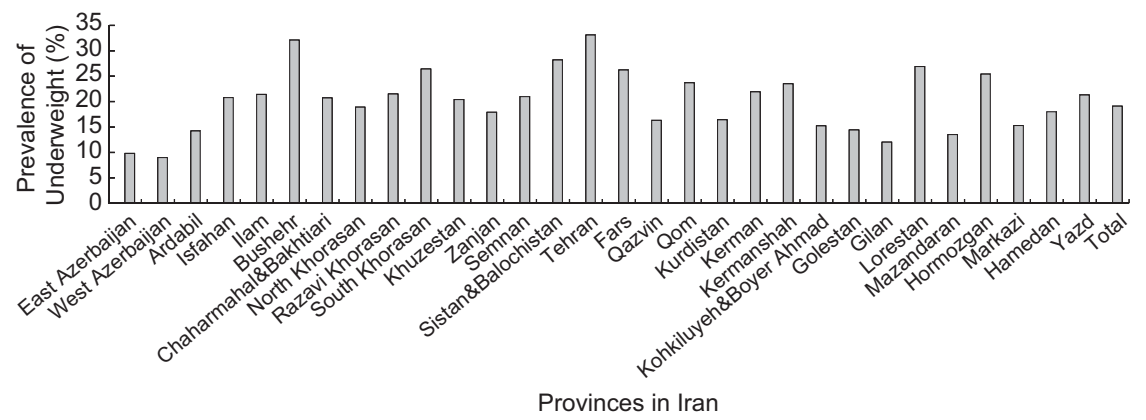

Fig. 2 Prevalence of underweight among 6-year-old children in different provinces in Iran, 2008 


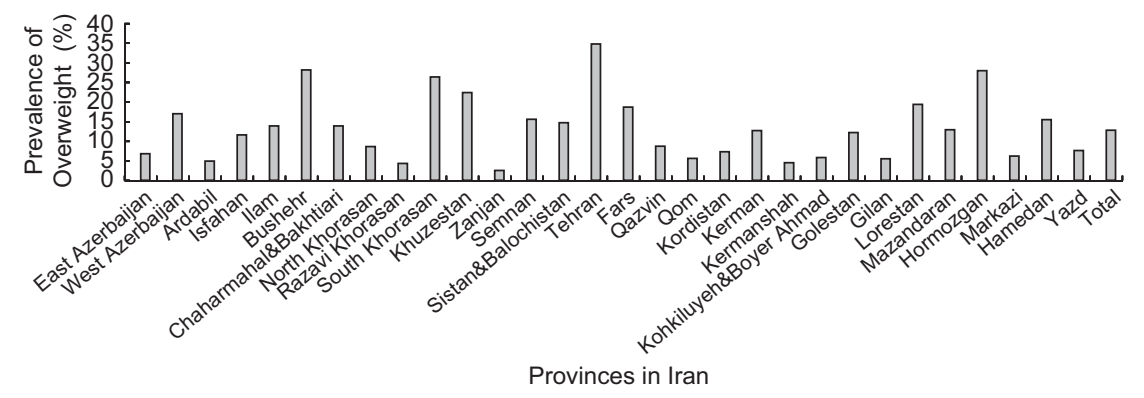

Fig. 3 Prevalence of overweight among 6-year-old children in different provinces in Iran, 2008

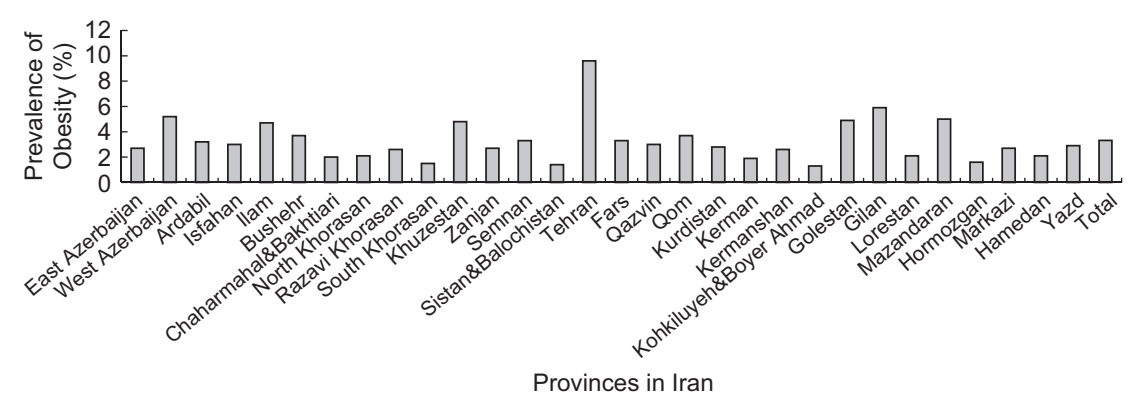

Fig. 4 Prevalence of obesity among 6-year-old children in different provinces in Iran, 2008

Overall, $16 \cdot 2 \%$ of children had a BMI more than 85 th percentile with a prevalence of $12 \cdot 8 \%$ for overweight and $3 \cdot 4 \%$ for obesity, respectively. Figures 3 and 4 show the considerably larger differences among the various provinces.

\section{Discussion}

To the best of our knowledge, the present study is one of the first national reports, not only from Iran but also from the Eastern Mediterranean region, providing information on weight disorders and short stature from the entire population of children at school entry. We documented marked differences in the regional distribution of short stature and weight disorders; this finding is consistent with other nationwide studies in developed and developing countries ${ }^{(13,14)}$.

The socio-environmental determinants of growth disorders have not been documented in the present survey; moreover, the populations of each province consist of various socio-economic groups and therefore the regional differences cannot be fully explained by the sociodemographic background of each province. As a general assumption, it can be said that the provinces with the highest prevalence of short stature were economically deprived; however, this prevalence was low in some other provinces with a nearly similar socio-economic situation. Other determinants explaining the residual variability in the prevalence of short stature will be determined in future studies. In a study of 600 Pakistani families of different socio-economic levels, the prevalence of short stature decreased with increasing income level ${ }^{(15)}$. In the present survey, our postulation is based on a general estimate of the economic situation of different provinces; future studies comparing the sociodemographic characteristics of the children's families might provide a more uniform association of economic features and short stature, as a marker of chronic undernutrition. Given the improvement in maternal and child nutritional status in recent decades in $\operatorname{Iran}^{(16)}$, the role of micronutrient deficiency should be determined in future studies on short stature in different provinces. It is noteworthy to mention that in the present survey, Fars province with middle-tohigh socio-economic status had a considerably higher prevalence of short stature; this is the region where the first case of zinc deficiency in man was reported in 1963 and increasing knowledge about the role of zinc in health and growth was documented afterwards ${ }^{(17)}$. Further studies conducted in 1990 confirmed the persistence of zinc deficiency in this area ${ }^{(18)}$; soil structure and climate characteristics might be related to this type of micronutrient deficiency in the region ${ }^{(19)}$. The socio-environmental determinants of short stature in Iranian children should be determined in future population-based studies.

Similar to short stature, underweight was more prevalent in provinces with low socio-economic status, although its prevalence was also considerably high in 
some provinces with better economic status including the capital city, Tehran. Although regional differences point to a predominance of environmental factors in explaining child undernutrition in poorer provinces, the large variability in socio-economic status of different provinces with a high prevalence of underweight demands nationwide studies on the determinants of this important public health problem.

It is worth mentioning that in addition to Tehran, overweight was most prevalent in Bushehr and Hormozgan, the provinces with simultaneous high prevalence of underweight and short stature. This is an expression of improper nutritional status of these provinces; moreover, it might reflect that the overweight status in these two economically deprived provinces, and other regions with similar socio-economic status, is because of the children's stunting, leading to an elevated BMI value. Usually, a combination of perinatal factors, notably maternal undernutrition, which leads to intra-uterine growth retardation, along with compromised lactation and infant feeding, results in stunting in early life. This pattern is usually followed by overfeeding of stunted children with highenergy foods, which in turn leads to rapid weight gain later in childhood ${ }^{(2,20,21)}$.

Given that the determinants of nutrition disorders vary across geocultural settings, information on local circumstances is essential to local as well as national planning and policy making. In the present study, obesity was more prevalent in Tehran and the northern provinces. This finding is consistent with the highest prevalence of overweight and obesity found among the adult population of these provinces studied in our previous nationwide survey ${ }^{(22)}$. In addition to genetic factors, this might be because of the obesogenic environment of the families. The aforementioned national study among adults revealed a positive association of sedentary lifestyle among the population living in these regions with generalized and abdominal obesity ${ }^{(22)}$. A study conducted among 6-11-year-old children in the provincial city of one of these northern provinces (Rasht in Gilan) confirmed the impact of familial factors on childhood overweight and genetic-environment interactions. It showed that children with overweight parents, children with more educated mothers and those skipping breakfast were more prone to overweight and obesity ${ }^{(23)}$.

Of special concern in the context of our study was the alarming prevalence of overweight and obesity in Tehran; overall, $44 \%$ of children had BMI $>85$ th percentile, i.e. $35 \%$ overweight and 9\% obesity. This considerably high prevalence is consistent with previous studies showing very high rates of overweight and obesity in different age groups in Tehran: a study in 2004-2007 reported a prevalence of $19 \cdot 4 \%$ of $\mathrm{BMI}>85$ th percentile in children aged 1-6 years ${ }^{(24)}$. This prevalence was reported to be $21 \%$ in 2004 among 11-16-year-old adolescents $^{(25)}$ and 25\% in 2004-2005 among adolescents aged 11-17 years ${ }^{(26)}$. Comparison of the prevalence of overweight and obesity in Tehranian adults showed that from 1998-1999 to 2000-2001, this prevalence increased from $75 \cdot 2 \%(42.5 \%$ overweight and $32 \cdot 7 \%$ obesity) to $86 \cdot 3 \%(46 \cdot 0 \%$ overweight and $40.3 \%$ obesity) in men and from $56 \cdot 5 \%(40 \cdot 0 \%$ overweight and $16 \cdot 5 \%$ obesity) to $60.3 \%(39 \cdot 5 \%$ overweight and $20 \cdot 8 \%$ obesity) in women $^{(27)}$. A cohort study in Tehran, which compared the prevalence of obesity among adults in 1999-2001, 2002-2005 and 2006-2008, revealed a steady increase in the prevalence of obesity from $15 \cdot 8 \%$ to $18.6 \%$ and $21.0 \%$ in men and from $31.5 \%$ to $37 \cdot 7 \%$ and $38.6 \%$ in women. The younger age group had a very high rate of increase in obesity, e.g. men aged 20-29 years had the highest rate of increased obesity $(23 \cdot 8 \%)$ and abdominal obesity $(88 \cdot 3 \%)^{(28)}$. Hence, it can be assumed that such a high prevalence of overweight and obesity among 6-year-old children is a critical concern leading to an emerging public health crisis of non-communicable diseases in young adulthood.

In addition to above-mentioned socio-economic diversity, the populations of various provinces in Iran have ethnic differences. The principal ethnic groups in Iran are Persian (51\%), Turk (24\%), Gilaki and Mazandarani (8\%), Kurd (7\%), Arab (3\%) and others (7\%). In the present survey, short stature and underweight, as indicators of malnutrition, were low in Zanjan and West Azerbaijan with Turk ethnicity, whereas these types of nutritional disorders were prevalent in Sistan and Balochistan, with a predominance of Baluch ethnicity, and Bushehr, with a predominance of Arab ethnicity. Although some of the variations can be explained by ethnic differences, other socio-environmental determinants may have a stronger impact on nutritional and growth disorders. For instance, Khuzestan and Bushehr, with a predominance of Arab ethnicity, had high rates of overweight; therefore, the coexistence of the highest rates of short stature, underweight and overweight in children living in Bushehr cannot be explained by their ethnic background. It might rather be a reflection of poor nutrition and improper dietary habits in this economically deprived region.

In general, our findings postulate that in young Iranian children, although undernutrition is still of crucial concern, the rapid transition to a Western profile with regard to overweight and obesity is significantly advanced.

This double burden of nutritional disorders is reported from many developing countries ${ }^{(29,30)}$, and this is one of the first national reports of the double burden of malnutrition in terms of short stature, underweight, overweight and obesity from the Eastern Mediterranean countries comprising all children at school entry. To the best of our knowledge, no previous study has been published from the developing countries about the data of a large sample size of $>850000$ children including the whole target population, thus without any selection bias. In spite of essential differences in the 
socio-environmental aspects of developing countries in various regions, as Shetty ${ }^{(31)}$ stated:

The changing environment is probably determining the altering scenario of child nutrition in developing societies, with rapid developmental transition and urbanization being responsible for the emerging problems of obesity and other metabolic disorders that are largely the result of the now well-recognized linkages between child undernutrition and early onset adult chronic diseases.

Similar to other developing countries, until now most of our national public health programmes and nutrition policies have focused on undernutrition and its effects on maternal-child survival as well as controlling infectious diseases. The health status of Iranian children has shown rapid improvement by reducing the mortality rate, full coverage of free vaccination and, in turn, eradication of major contagious disease, free services for infant and child health care in the well-established network of health centres and a structured referral system and mandatory free screening of all children at school entry. However, the findings of the present survey, which is part of the aforementioned screening programme at school entry, make clear that, in addition to these successful health policies for undernutrition and communicable diseases, it is necessary to pay more attention to the prevention and control of other aspects of malnutrition, i.e. overweight, and, in turn, risk factors of non-communicable diseases. Developing countries are facing an emerging epidemic of noncommunicable diseases. This is of special importance in the Middle East because it would have the highest burden of diabetes and related complications, notably CVD, in the next decade ${ }^{(32)}$. The findings of the present survey call for rapid evidence-based policy development and interventions to address the dual problem of underweight and overweight among young children. Given that the risk factors of non-communicable diseases are not limited to overweight children ${ }^{(33,34)}$, controlling both types of malnutrition, i.e. underweight and overweight, might have a long-term impact on the burden of chronic diseases in low- and middle-income countries. In fact, most educational and interventional programmes targeting a healthy lifestyle for children of many developing communities, including ours, are considered for school-aged children and adolescents and mostly through school health policies. The findings of the present survey among young children before school entrance make it obvious that a healthy lifestyle, notably in terms of healthy nutrition and physical activity, should be addressed in primary-care settings and child health-care services. Moreover, public awareness should be increased and subjects related to prevention and control of underweight and overweight should be increased in the curriculum of health professionals.

We should consider the findings of the present study under its limitations and strengths: the main limitation of the present study is its cross-sectional nature. Moreover, considering the very large sample size of the study, we could not document details of socio-environmental and lifestyle determinants of the dual growth disorders among the children under study. The main strengths of the study are its nationwide coverage of all school-entry children, as well as its novelty in reporting the prevalence of short stature, underweight, overweight and obesity among young children as one of the first national surveys in the Eastern Mediterranean region.

\section{Conclusion}

The coexistence of the high prevalence rates of short stature and underweight along with overweight and obesity in children of the same community warrants a multi-faceted national policy with evidence-based local programmes to address the double burden of nutritional disorders. Such planning needs a comprehensive surveillance system and a centralized data registry for children's nutrition and growth. Given that the determinants of growth disorders vary across geocultural settings, information on local circumstances as well as children and families' dietary and physical activity habits is essential to local and national planning and policy making.

\section{Acknowledgements}

The present study was part of a national screening study supported by the Ministry of Health and Medical Education and the Ministry of Education and Training. The authors declare that they have no conflict of interest. M.E.M., M.A.A., H.Z., M.D., T.A., G.A. and S.K. contributed in conducting the study; R.K., P.M. and P.P. prepared the manuscript. All authors have read and confirmed the manuscript. The authors forward their sincere thanks to the large team working for the present study at national level.

\section{References}

1. de Pee S, Brinkman HJ, Webb P et al. (2010) How to ensure nutrition security in the global economic crisis to protect and enhance development of young children and our common future. J Nutr 140, 138S-142S.

2. Kelishadi R (2007) Childhood overweight, obesity, and the metabolic syndrome in developing countries. Epidemiol Rev 29, 62-76.

3. Olivieri F, Semproli S, Pettener D et al. (2008) Growth and malnutrition of rural Zimbabwean children (6-17 years of age). Am J Phys Anthropol 136, 214-222.

4. Jones LL, Griffiths PL, Adair LS et al. (2008) A comparison of the socio-economic determinants of growth retardation in South African and Filipino infants. Public Health Nutr 11, 1220-1228.

5. Bomela NJ (2009) Social, economic, health and environmental determinants of child nutritional status in three Central Asian Republics. Public Health Nutr 12, 1871-1877. 
6. Monteiro CA, Conde WL \& Popkin BM (2002) Is obesity replacing or adding to undernutrition? Evidence from different social classes in Brazil. Public Health Nutr 5, 105-112.

7. Onis M \& Blossner M (2000) Prevalence and trends of overweight among preschool children in developing countries. Am J Clin Nutr 72, 1032-1039.

8. Ghassemi H, Harrison G \& Mohammad K (2002) An accelerated nutrition transition in Iran. Public Health Nutr 5, 149-155.

9. Kelishadi R, Ardalan G, Gheiratmand R et al:; Caspian Study Group (2008) Thinness, overweight and obesity in a national sample of Iranian children and adolescents: CASPIAN Study. Child Care Health Dev 34, 44-54.

10. Kelishadi R, Ardalan G, Gheiratmand R et al:; CASPIAN Study Group (2007) Association of physical activity and dietary behaviours in relation to the body mass index in a national sample of Iranian children and adolescents: CASPIAN Study. Bull World Health Organ 85, 19-26.

11. Kuczmarski RJ, Ogden CL \& Grummer-Strawn LM (2000) CDC growth charts: United States. Adv Data 314, 1-27.

12. Wit JM, Clayton PE, Rogol AD et al. (2008) Idiopathic short stature: definition, epidemiology, and diagnostic evaluation. Growth Horm IGF Res 18, 89-110.

13. Zimmermann MB, Gübeli C, Püntener C et al. (2004) Overweight and obesity in 6-12 year old children in Switzerland. Swiss Med Wkly 134, 523-528.

14. El-Hazmi MA \& Warsy AS (2002) A comparative study of prevalence of overweight and obesity in children in different provinces of Saudi Arabia. J Trop Pediatr 48, 172-177.

15. Hakeem R (2001) Socio-economic differences in height and body mass index of children and adults living in urban areas of Karachi, Pakistan. Eur J Clin Nutr 55, 400-406.

16. Djazayery A (2004) Regional overview of maternal and child malnutrition: trends, interventions and outcomes. East Mediterr Health J 10, 731-736.

17. Prasad AS (2001) Recognition of zinc-deficiency syndrome. Nutrition 17, 67-69.

18. Lankarani S \& Musaiger AO (1991) The state of nutrition in Fars, Iran: a review. Nutr Health 7, 135-142.

19. Karami M, Afyuni M, Khoshgoftarmanesh AH et al. (2009) Grain zinc, iron, and copper concentrations of wheat grown in central Iran and their relationships with soil and climate variables. J Agric Food Chem 57, 10876-10882.

20. Shapira N (2008) Prenatal nutrition: a critical window of opportunity for mother and child. Womens Health (Lond Engl) 4, 639-656.

21. Crawford MA (2008) The elimination of child poverty and the pivotal significance of the mother. Nutr Health 19, 175-186.
22. Kelishadi R, Alikhani S, Delavari A et al. (2008) Obesity and associated lifestyle behaviours in Iran: findings from the First National Non-communicable Disease Risk Factor Surveillance Survey. Public Health Nutr 11, 246-251.

23. Maddah M \& Nikooyeh B (2009) Factors associated with overweight in children in Rasht, Iran: gender, maternal education, skipping breakfast and parental obesity. Public Health Nutr 23, 1-5.

24. Ataei N, Hosseini M \& Iranmanesh M (2009) The relationship of body mass index and blood pressure in Iranian children $<7$ years old. J Trop Pediatr 55, 313-317.

25. Mohammadpour-Ahranjani B, Rashidi A, Karandish M et al. (2004) Prevalence of overweight and obesity in adolescent Tehrani students, 2000-2001: an epidemic health problem. Public Health Nutr 7, 645-648.

26. Moayeri H, Bidad K, Aghamohammadi A et al. (2006) Overweight and obesity and their associated factors in adolescents in Tehran, Iran, 2004-2005. Eur J Pediatr 165, 489-493.

27. Azizi F, Azadbakht L \& Mirmiran P (2005) Trends in overweight, obesity and central fat accumulation among Tehranian adults between 1998-1999 and 2001-2002: Tehran lipid and glucose study. Ann Nutr Metab 49, 3-8.

28. Hosseinpanah F, Barzin M, Sarbakhsh Eskandary $\mathrm{P}$ et al. (2009) Trend of obesity and abdominal obesity in Tehranian adults: a cohort study. BMC Public Health 9, 426.

29. Duran P, Caballero B \& de Onis M (2006) The association between stunting and overweight in Latin American and Caribbean preschool children. Food Nutr Bull 27, 300-305.

30. Jinabhai CC, Taylor M \& Sullivan KR (2003) Implications of the prevalence of stunting, overweight and obesity amongst South African primary school children: a possible nutritional transition? Eur J Clin Nutr 57, 358-365.

31. Shetty P (2009) Community-based approaches to address childhood undernutrition and obesity in developing countries. Nestle Nutr Workshop Ser Pediatr Program 63, 227-254.

32. Wild S, Roglic G, Green A et al. (2004) Global prevalence of diabetes: estimates for the year 2000 and projections for 2030. Diabetes Care 27, 1047-1053.

33. Kelishadi R, Cook SR, Motlagh ME et al. (2008) Metabolically obese normal weight and phenotypically obese metabolically normal youths: the CASPIAN Study. $J$ Am Diet Assoc 108, 82-90.

34. Ramachandran A, Snehalatha C, Yamuna A et al. (2007) Insulin resistance and clustering of cardiometabolic risk factors in urban teenagers in southern India. Diabetes Care 30, 1828-1833. 